\title{
Word Knowledge Acquisition, Lexicon Construction and Dictionary Compilation
}

\author{
Antonio Sanfilippo* \\ SHARP Laboratories of Europe litd. \\ Oxford Science Park, Oxford OXA AGA, UK \\ antonioosharp.co.uk
}

\begin{abstract}
We describe an approach to semiautomatic lexicon development from machine readable dictionaries with specific reference to verbat diatheses, envisaging ways in which the results obtained citl be used to guide word classification in the construction of dictionary databases.
\end{abstract}

\section{Introduction}

The acquisition and representation of lexical knowledge from machine-readable dictionaries and text corpora have increasingly become major concerns in Conputational Lexicography/Lexicology. While this trend was essentially set by the need to maximize costeflectiveness in building large scale Lexical Kuowledge Bases for NLP' (LKBs), there is a clear sense in which the construction of such knowledge bases also caters to the demand for better dictionaries. Currently available dictionaries and thesauri provide an undoubtedly rich source of lexical information, but often omit or neglect to make explicit salient syntactic and scmantic properties of word entries. For example, it is well known that the same verb sense can appear in a variety of subcategorization frames which can be related to one another through valency alternations (diatheses). Some dictionaries provide subcategorization information by means of grammar codes, as shown below for the "sail" sense of the verb dock in IJDOCF - Longman's Dictionary of Contemporary linglish (Procter, 1978).

(1) $\operatorname{dock}^{4} v[\mathrm{~T} 1 ; \mathrm{I}(0:(a t)], \ldots$

The codes $[\mathrm{T} 1 ; \mathrm{I} 0:(a t)]$ indicate that the verb can be either transitive or intransitive with the possible artdition of an oblique complement introduced by the preposition at:

(2) a. [T1 (at)]: Kim docked his ship (at Glasgow)

b. [I0 $(a t)]$ : The ship docked (at (ilasgow)

Unfortunately, an indication of diatheses which relate the various occurrences of the verb to one another is rarely provided. Consequently, if we were to use the grammar code information found in I,DOCE to create verb entries in an IKKB by automatic conversion we would construct four seemingly unrelated entries for the verb dock (see \$3). Inadequacies of this kind may be redressed through semiantomatic techniques

"The research reported in this paper was carried out within the $A C Q U I J F$ project. I am indebted to Ted Briscoe, Ann Copestake and Pete Whitelock for helpful comments. which make it possible to supply information concerning arnenability to diathesis alternations so as to avoid expanding distinct entries for related uses of the same verb. This practice would allow us to develop an I,KB from diclionary databases which offers a more complete and linguistically refined repository of lexical information than the source databases. Such an I,KB would be used to generate lexical components for NISP systems, and could also be integrated into a lexicographer's workstation to guide word classification.

\section{The $\Lambda C Q U I L E X$ Iexicon Development Environment}

Our points of departure are the tools for lexical acquisition and knowledge representation developed as part of the ACQUIILX project ("The Acquisition of Lexical Knowledge for NLP' Systems').

The $\Lambda$ CQUIJPX I.exicon Development Finvironment uses typed graph unification with inheritance as its lexical representation language (for details, see Copestake (1992), Sanfilippo \& Poznaúski (1992), and papers by Copestake, de Paiva and Sanfilippo in Briscoe et al. (1993)). It allows the user to define an inheritance hierarchy of types with associated restrictions expressed in terms of attribute-value pairs as shown in lig 1, and to create lexicons where such lypes are used to create lexical templates which encode word-sense specific information extracted from MRI)s such as the one in lig 2. (Bold lowercase is used for types, caps for attributes, and boxes enclosing types indicate total omission of attribute-value pairs. Details concerning the encolling of verb syntax and semanties can be found in Sanfilippo (1993).)

Feature Structure (FS) descriptions of word senses such ats the one in Fig 2 are created semiautomatically throngh a progran which converts syntactic and

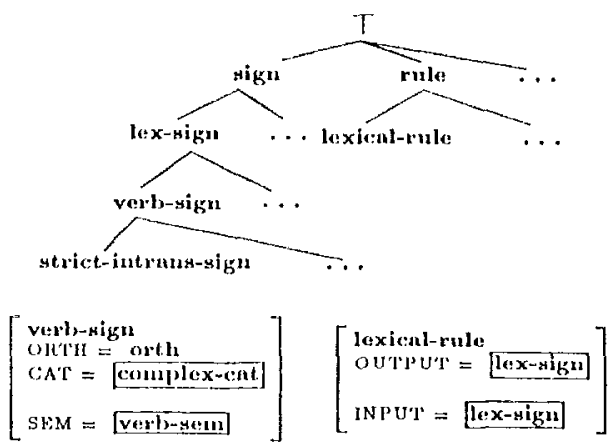

Figure 1: Type Hierarchy \& Constraints (fragment). 


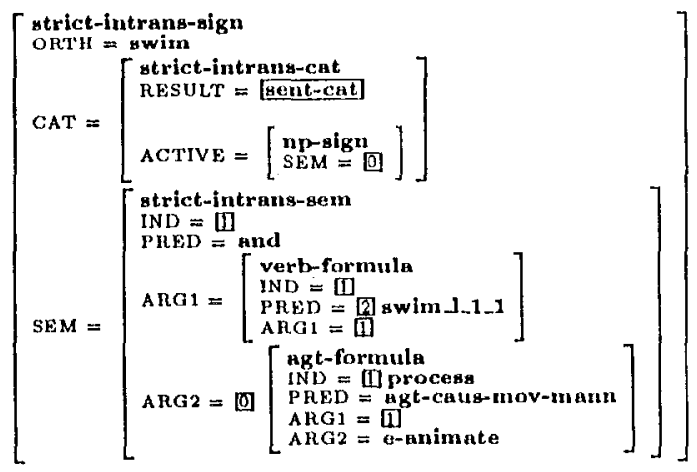

Figure 2: LKB Entry for swim (simplified).

semantic specifications encoded in MRDs into LKB types. For example, the choice of LKB types used in the characterization of the verb swim above was induced from the syntactic and semantic codes found in LDOCE and the Longman Lexicon of Contemporary English (LLOCE, McArthur 1980). In LDOCE, the first sense of the verb swim is marked as a strict intransitive verb ([I0]) whose subject is animate ((box - - $)$ ); in LLOCE, the same verb sense is semantically classified as a movement verb with manner of motion specified (M19):

swim $1(1)$
LDOCE
LLOCE

[I0] (box - - - o) ...

M19 - Particular ways of moving

The MRD-to-LKB equivalences induced by the conversion algorithm are as shown in (1) where agt-causemove-manner indicates that the subject participant relation implies self-induced movement with manner specified.

(4) [IO

$$
\begin{aligned}
& \text { 10] } \rightarrow \text { strict-intrans-sign } \\
& \text { (box - - 0) } \rightarrow \quad[\text { CAT: ACTIVE : SEM : ARG } 2= \\
& \text { o-animute] } \\
& \text { M19 } \rightarrow \text { (CAT:ACTIVF: SEM: PRI) }= \\
& \text { [10], M19 } \rightarrow \quad \text { [SEM : IND = process] }
\end{aligned}
$$

\section{Verbal Diatheses and Lexical Acquisition}

In the example discussed above, MRD-to-LKI conversion is relatively straightforward: a single LKB entry is created for swim since a single grammar code is found in the MRD sources used. Where a verb-sense entry gives more than one grammar code, however, the question arises whether or not each grammar code should be mapped into a distinct LKB entry. For example, the codes given in LDOCE for the verb dock (see (1)) could potentially be used to derive four LKB verb entries:
(5)
LKB TYPF
EXAMPLI:
a. strict-trans-sign
Kim docked the boat
b. obl-trans-sign
Kim docked the boat
at Southampton
c. strict-intrans-sign The boat docked
d. obl-intrans-sign The boat docked at
Sonthampton

Notice, however, that in this case the creation of four distinct $L K B$ entries is unnecessary insofar as the use of the verb exemplified in (5b) contains enough information to derive the remaining uses of the verb through lexical rules which progressively reduce the verb's valency by dropping the subject and/or prepositional argument(s). Such a step would be linguistically motivated in that it establishes a clear link between alternative uses of the same verb sense. Moreover, compact representation of verb use extensions is desirable from an engineering perspective as it reduces the size of the lexicon, allowing verb use expansion to be delayed till parsing time. 'This practice can be made to facilitate the resolution of lexical ambiguity by enforcing selective application of lexical rules (Copestake \& Briscoe, 1994).

Compact representation of verb use extensions due to valency alternations requires that a note of all applicable lexical rules be made in each kernel entry. In choosing obl-trans-sign as the LKB type for dock, for example, specifications would be added saying that the verb is amenable to the causative-inchoative alternation relating agentive and agentless uses $((5 \mathrm{a}, \mathrm{b})$ vs. $(5 c, d))$, and the path alternation pertaining to the omission of the prepositional argument $((5 \mathrm{a}, \mathrm{c})$ vs. $(5 \mathrm{~b}, \mathrm{~d}))$. In addition, the palh alternation would have to be specified as to whether it preserves amenability to a telic interpretation (accomplishment or achievement) of the event described by the verb or not. For example, the omission of the goal argument for a verb such as lrive, push or carry induces an atelic (process) interpretation as indicated by incompatibility with a terminative adverbial

(6) a. Joln drove his car to London in one hour

b. John drove his car (*in one hour)

Within a (partial) decompositional approach to verb semantics ('Talmy, 1985; Jackendoff, 1990; Sanfilippo, 1993; Sanlilippo ef al, 1992)), this contrast can be explained with reference to the meaning component path. In (6a), the goal argument (to London) fixes a final bound for the path along which the driving event. takes place. Assuming that the compositional meaning of the sentence involves establishing a homomorphism between the event described by the verb and the path along which such an event takes place (Dowty, 1991; Sanfilippo, 1991), it follows that with an unbounded path (c.g. (6b)) only a process interpretation is possible, whereas with a bounded path (e.g. (6a)) a telic interpretation is more likely. By contrast, the omission of the goal argument with verbs such as deliver, bring, dock and send does not inhibit amenability to a telic interpretation, c.g.

(7) We can deliver the goods (to your door) in one hour 
Our aim, then, was to capture regularities across distinct uses of the same verb sense by relating the subcategorization frames relative to these uses via regular syntactic and semantic changes. To assess the feasibility of this approach, we augmented the MRD-to-LKB conversion code with facilities which make it possible to infer amenability to specific diathesis alternations from occurrence of multiple grammar codes and their associated semantic codes in the MRIDs. To improve on the informational content of LDOCE grammar codes, we used an intermediate dictionary semiautomatically derived from IJDOCE (LDOCE_Inter) where the subcategorizalion information inferrable from grammar codes and other orthographic conventions was made more explicit (Boguraev \& Briscoe, 1989; Carroll \& Grover, 1989). Semantic information about verb classes was obtained by mapping across L,J)OCF and LLOCE so as to angment LDOCE queries with thesaurus information, i.e. semantic codes (Sanfilippo \& P'oznański, 1992).

Syntactic and semantic information relalive to verb senses was extracted through special finnctions which operate on pointers to dictionary entries. 'The extracted info was used to generate lis representations of word senses. The conversion process was carried out in such a way that whenever multiple subcategorization frames were found in association wilh a verb sense, only those which could not be derived via diathesis alternation were expanded into LKB entries. For example, the LDOCE_Inter entry for dock gives four subcategorization frames:

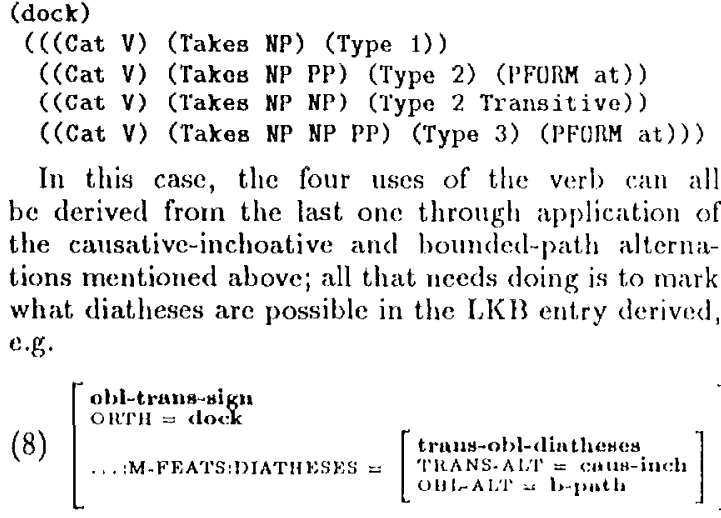

The algorithm which guides this process checks whether information regarding diathesis alternations can be inferred from dictionary entries in the MRI) sources or must be manually supplied. In performing this check, subcategorization options relative to a given verb sense which can be inferred from a more informative subcategorization frane are ignored. 'This technique was successfully employed in semiautomatic derivation of lexicons for 360 movernent verbs yielding over 500 additional possible expansions by application of lexical rules.

\section{Verbal Diatheses and Knowledge Representation}

To encode anenability to verbal diatheses, the feat ture bIaTumses was introduced as an extension of the morphological features associated with verbs (see (8)). This feature takes as value the type alternations which is in turn defined as having a variety of specialized types according to which diathesis alternations are aclmissible for each choice of verb type (e.g. intransitive, transitive, ditransitive), as shown in rigure 3 (see next page). The following table provides examples of the diatheses referred to in Fig 3.

\begin{tabular}{|c|c|}
\hline DIA'TUESIS & BXAMPLE \\
\hline caus-inch & $\begin{array}{l}\text { Kim broke the glass vis. } \\
\text { the glass broke }\end{array}$ \\
\hline middle & $\begin{array}{l}\text { Kim scares Sally vs. } \\
\text { Sally scarcs easily }\end{array}$ \\
\hline indef-ohj & $\begin{array}{l}\text { John ale a sandwich vs. } \\
\text { John ale }\end{array}$ \\
\hline def-ol,j & $\begin{array}{l}\text { John did not notice the sign vs. } \\
\text { John did not notice. }\end{array}$ \\
\hline recip) & $\begin{array}{l}\text { Kim met Bill vs. } \\
\text { Kim and Bill met }\end{array}$ \\
\hline puss & $\begin{array}{l}\text { Bill read the Guardian vs. } \\
\text { The Guardian was read by Bill }\end{array}$ \\
\hline b-path & $\begin{array}{l}\text { Kim returned the book to Sue vs. } \\
\text { Kim returned the book } \\
\text { Kim camo away vs. } \\
\text { Kim came (particle alternation) }\end{array}$ \\
\hline u-path & $\begin{array}{l}\text { Kim swam across the river vs. } \\
\text { Kim swam } \\
\text { Kim walked away vs. } \\
\text { Kim walked (particle alternation) }\end{array}$ \\
\hline to/fore & $\begin{array}{l}\text { John brought a book to/for Sue vs. } \\
\text { John brought Sue a book }\end{array}$ \\
\hline
\end{tabular}

Diathesis alternations are enforced by means of lexical rules which, on par with all other information structures in the LKI, are hicrarchically arranged, as shown in Fig 4 with relerence to the bound and unbound path alternations for intransitive verbs. Lexical rules

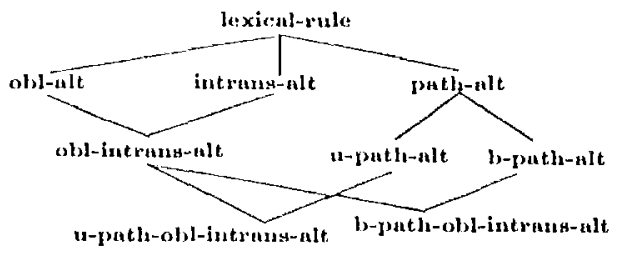

Figure 4: Lexical Rule Ilierarchy (fragment).

enforcing diathesis alternations may involve a variety of syntactic, semantic and orthographic changes. For example, the n-path-obl-intrans-alt rule shown in lig 5 below tikes as inpul an l'S of lype obl-intranssign which represents a verb describing a non-stative eventuality (dyn-eve) whose subject participant (with semantics 目) is implied as moving along a directed path (th-move-dir) the endpoint of which is specified by the oblique argument (pp-sign), e.g. the use of suim in Kim swam across the river. The ontput is an IS representing a strict intransitive verb (strictintrans-sign) which describes a process and whose subject participant is like that of the input with the directed path specification removed (th-move instead of th-move-dir), e.g. swim in Kim swam).

\section{Using the LKM to Guide Dictionary Compilation}

There are at least two ways in which an LKB such as the one developed in $A C Q U L L$ L offers the means to 


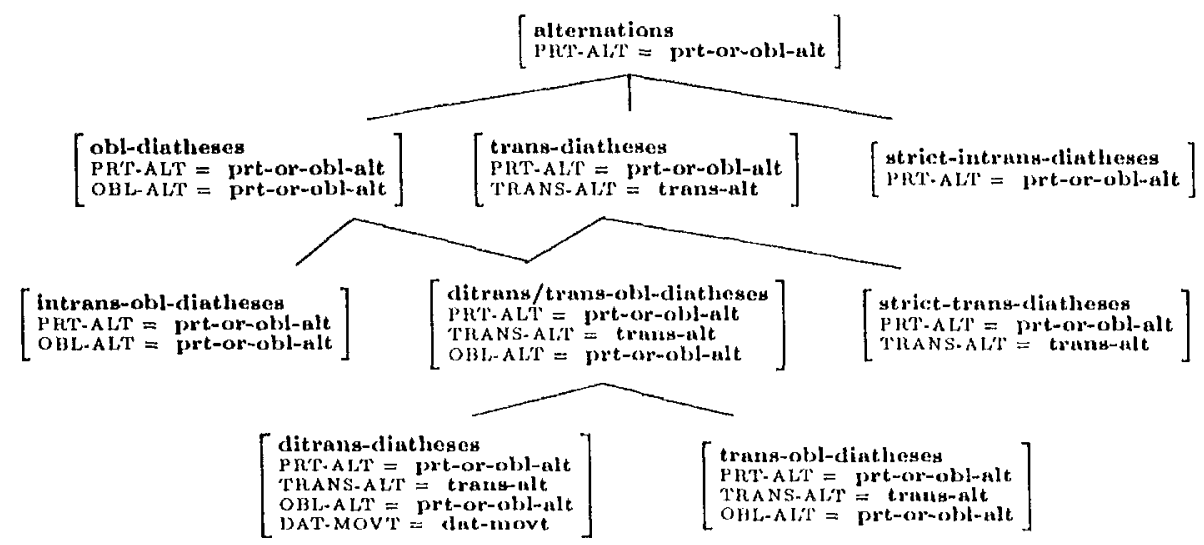

trans-alt $\subseteq$ caus-inch, middle, indef-obj, def-obj, recip, pass

prt-or-obl-ult [ b-path, u-path

dat-movt $\subseteq$ to, for

Figure 3: Verbal Diatheses Ilierarchy

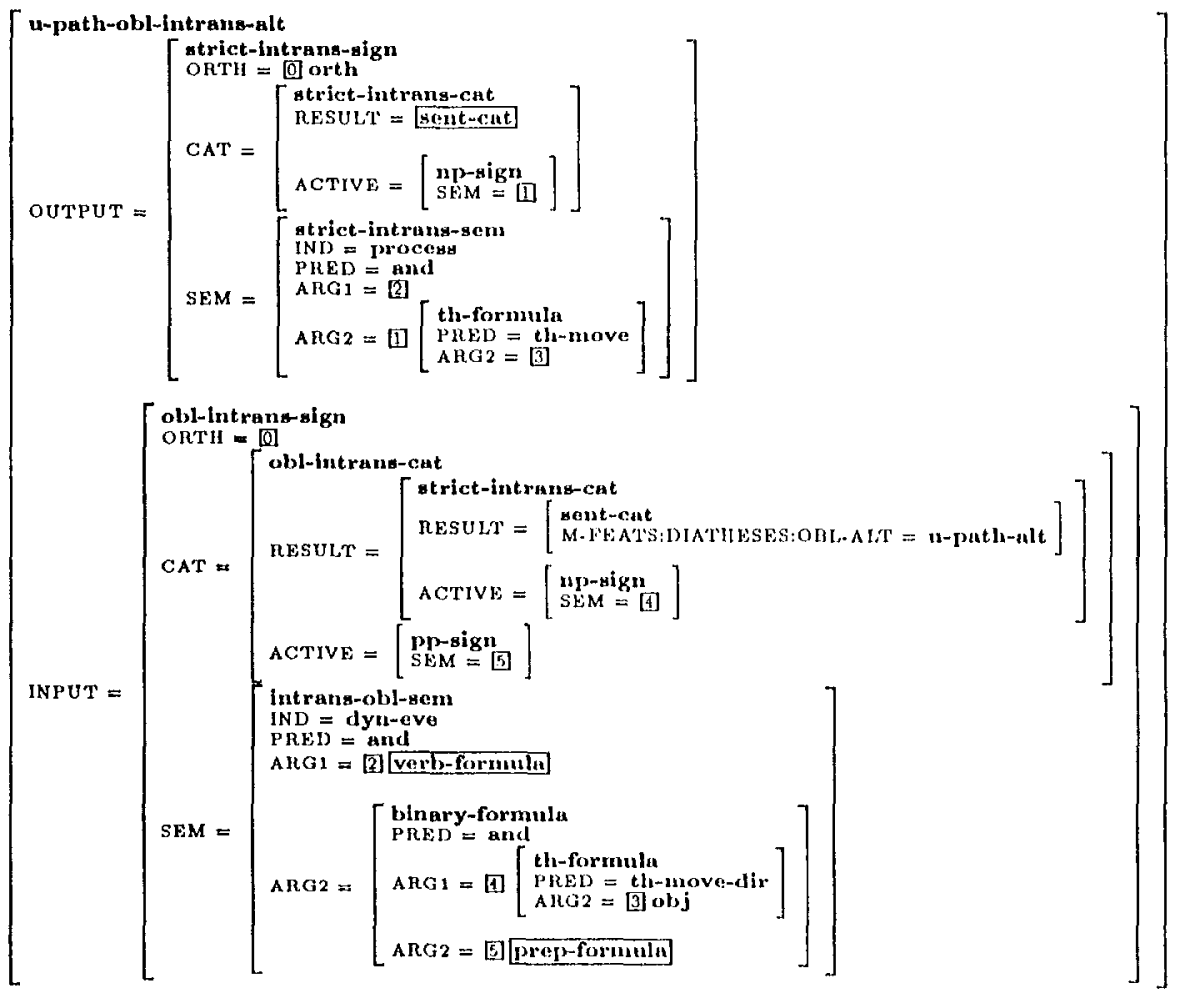

Figure 5: The "unbounded path" lexical rule for intransitives 


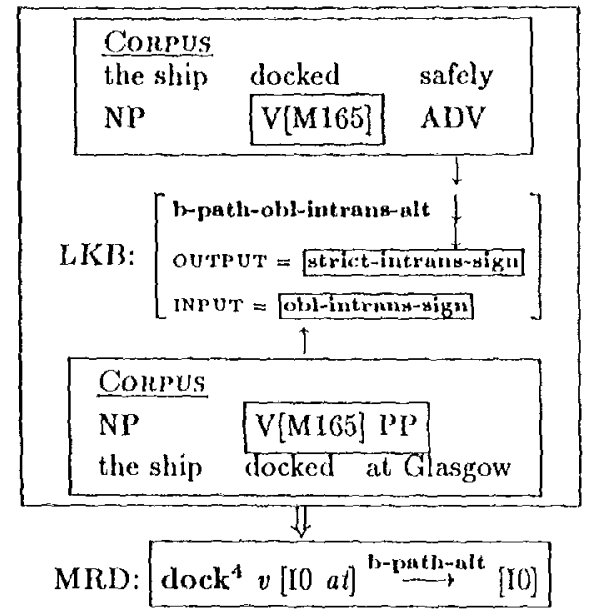

Figure 6: T'BA

facilitate word classification in the compilation of new lexical databases.

Pirst, the links between LKl3 types and dictionary entries established in the conversion stage can be used to run consistency checks on the MR,I) sources and to supply missing information or correct errors. 'This offers an efficient and cost-effective way of generating improved versions of the same dictionary.

Second, the types associated with specific word classes can be made to guide lexical aquisition from corpora when creating new dictionaries. It is now widely recognized that corpora are indispensable in the acquisition of lexical information relating to issues of usage such as the range and frequency of different patterns of syntactic realization. The availability of software tools for partial analysis of texts (e.g. morphological and semantic tagging, phrasal parsing etc.) has increased significantly the ntility of corpora in lexical acquisition by providing ways to structure the information contained in them (see Briscoe (1991) and references therein). Further advances yet can be made by using LKII types to classify words in text corpora. Suppose, for example, we linked the input and output of lexical rules to semantically tagged subeategorization frames extracted from bracketed corporit (Poznański \& Sanfilippo, 1993). As inclicated in Fig 6, this would allow us to assess which alternations might be of interest in establishing regular verb sense/usage shifts. Such an assessment would provide an effective way to drive verb categorization from corpora in the domain of valency alternations.

\section{Final Remarks}

A key element in our approach to lexical acquisition and representation of verbal diathesis concerns the use of semantics constraints in formulating MIRI queries and characterizing FS descriptions. This practice ensures that the results achieved in this work for motion verbs can be suitably extended to other semantic verb classes. For example, the class of verbs which undergo "extraposition" - e.g. That Kim lefl early bathers Sue vs. It bothers Sue that Kim left early - can be identified by using semantic constraints on MRI queries which identify psychological verbs with stimm- lus subject such as bother, please, etc. (Sanfilippo \& Poznatiski, 1992). This approach provides an effective way of employing semiautomatic extraction of information from MRDs for lexicon construction, and it facilitates word classification from text corpora when compiling new dictionary databases.

\section{References}

Briscoe, T. (1991) Lexical Issues in Natural Language Processing. In Klein, F. \& F. Veltman (eds.). Natural Language and Speech, Springer-Verlag, 39-68.

Briscoe, T., $\Lambda$. Copestake and V. de Paiva (1903) Defaull Inherilance willin Unificalion-Based $A p$ proaches to the lexicon, CUP.

Boguraev, B. \& T. Uriscoe (1989) Utilising the I,DOCF Grammar Codes. In Boguraev, 13. \& Briscoe, 'T. (eds.) Computational Lexicoyraphy for Natural Language Jrocessing. Longman, London.

Carroll, J. \& C. Grover (1989) The Derivation of a Large Computational Lexicon for Englisls from LDOCP. In Boguraev, B. \& Briscoe, T'. (eds.) Comtpulational Lexicography for Nalural Language Processing. Longman, London.

Copestake, A. (1992) The ACQUILFX LKB: Representation Issues in Semi-Automatic Acquisition of large lexicons. In Proceedings of the 3rd Conference on Applicd Natural Ianguaye Processing.

Copestake, $\AA$. and T. Briscoe (1994) Semi-productive Polysemy and Sense Pxtensions. Ms. Computer Laboratory University of Cambridge, Xerox Park (Menlo Park) and Rank Xerox Research Laboratory (Grenoble).

Dowty, D. (1991) Thematic Proto-Roles and Argument Selection. Languatge 67, pp. 547-619.

Jackendoft, R. (1990) Semantic Strtectures. MT'l Press, Cimbridge, Mass.

McArthur, 'T'. (1981) Longman Lexicon of Contemporary English. Longman, London.

Poznaríski \& Sanfilippo (1993) Detecting Dependencies between Semantic Verb Subclasses and Subcategorization Frames in Iext Corpora. In B. Bogurace \& J. P'ustejovsky (eds) Acquisition of Lexical Knowledge from that, Procecdings of a SICLEX workshop ACL, 93 , Olio.

Procter, P. (1978) Longman Dicionary of Contemporary linglish. Longman, London.

Santilippo, A. (1991) Thematic and Aspectual Information in Verb Semantics. Belgian Journal of hinguistics, 6 .

Sunfilippo, A. (1993) LKB lincoding of lexical Knowledge. In Briscoe, 'P., $A$. Copestake and $V$. de Paiva (eds.).

Sanfilippo, $A$ \& V. Poznanski (1992) T'he Accuisition of Lexical Knowledge from Combined MachincReadable Dictionary Sources. In Proceedings of the Sol Conference on Applied Nalural Language Pro. cessing, 'Irento.

Sanfilipjo, A., T. Briscoe, A. Copestake, M. Martí, M. Taulé and $\Lambda$. Alonge (1992) 'Translation Vquivalence and Jexicalization in the $\Lambda$ CQUILEX IKB. Proceedings of 'I'M1-92, Montreal, Canada.

'Talny, 1. (1985) Lexicalization Patterns: Semantic Structure in Lexical liorm. In Shopen, 'T. (ed) Languagc Typology and Syntactic Descriplion S. Crammatical Categoriesv and the Lexicon, CUP'. 